\title{
Reward Region Responsivity Predicts Future Weight Gain and Moderating Effects of the TaqIA Allele
}

\author{
Eric Stice, ${ }^{1}$ Kyle S. Burger, ${ }^{2}$ and Sonja Yokum ${ }^{1}$ \\ ${ }^{1}$ Oregon Research Institute, Eugene, Oregon 97403 and ${ }^{2}$ University of North Carolina at Chapel Hill, Department of Nutrition, CB 7461, Chapel Hill, North \\ Carolina 27599
}

\begin{abstract}
Because no large prospective study has investigated neural vulnerability factors that predict future weight gain, we tested whether neural response to receipt and anticipated receipt of palatable food and monetary reward predicted body fat gain over a 3-year follow-up in healthy-weight adolescent humans and whether the TaqIA polymorphism moderates these relations. A total of 153 adolescents completed fMRI paradigms assessing response to these events; body fat was assessed annually over follow-up. Elevated orbitofrontal cortex response to cues signaling impending milkshake receipt predicted future body fat gain $(r=0.32)$, which is a novel finding that provides support for the incentive sensitization theory of obesity. Neural response to receipt and anticipated receipt of monetary reward did not predict body fat gain, which has not been tested previously. Replicating an earlier finding (Stice et al., 2008a), elevated caudate response to milkshake receipt predicted body fat gain for adolescents with a genetic propensity for greater dopamine signaling by virtue of possessing the TaqIA A2/A2 allele, but lower caudate response predicted body fat gain for adolescents with a genetic propensity for less dopamine signaling by virtue of possessing a TaqIA A1 allele, though this interaction was only marginal $[p$-value $<0.05$ corrected using voxel-level familywise error rate $(\mathrm{pFWE})=0.06$ ]. Parental obesity, which correlated with TaqIA allele status (odds ratio $=2.7$ ), similarly moderated the relation of caudate response to milkshake receipt to future body fat gain, which is another novel finding. The former interaction implies that too much or too little dopamine signaling and reward region responsivity increases risk for overeating, suggesting qualitatively distinct reward surfeit and reward deficit pathways to obesity.
\end{abstract}

Key words: obesity; prospective fMRI; reward sensitivity; weight gain

\section{Significance Statement}

Because no large prospective study has investigated neural vulnerability factors that predict future weight gain we tested whether neural response to receipt and anticipated receipt of palatable food and monetary reward predicted body fat gain over 3-year follow-up in healthy-weight adolescent humans and whether the TaqIA polymorphism moderates these relations. Elevated reward activation in response to food cues predicted future body fat gain. Elevated reward response to food receipt predicted body fat gain for adolescents with a TaqIA A2/A2 allele and lower reward response predicted body fat gain for those with a TaqIA A1 allele. Results imply that too much or too little dopamine signaling and reward region responsivity increases risk for overeating.

\section{Introduction}

The prevalence of obesity has risen worldwide and is credited with 2.8 million premature deaths annually (World Health Organization, 2013), but treatments rarely result in lasting weight

\footnotetext{
Received Aug. 28, 2014; revised June 1, 2015; accepted June 9, 2015.

Author contributions: E.S. designed research; K.S.B. and S.Y. performed research; K.S.B. and S.Y. analyzed data; E.S., K.S.B., and S.Y. wrote the paper.

Support for this work was provided by National Institutes of Health Grant DK-080760. We thank the Lewis Center

for Neuroimaging at the University of Oregon for their assistance in imaging for this investigation.

The authors declare no competing financial interests.

Correspondence should be addressed to Eric Stice, Oregon Research Institute, 1776 Millrace Drive, Eugene, OR

97403. E-mail: estice@ori.org.

DOI:10.1523/JNEUROSCI.3607-14.2015

Copyright $\odot 2015$ the authors $\quad 0270-6474 / 15 / 3510316-09 \$ 15.00 / 0$
}

loss (Turk et al., 2009). An improved understanding of neural vulnerability factors that predict future weight gain should inform the design of more effective treatments. Palatable food intake and cues increase activation in regions implicated in reward, including the striatum, midbrain, amygdala, and orbitofrontal cortex (OFC), and cause dopamine (DA) release in the dorsal striatum that scales with meal pleasantness ratings and caloric density of the food (Small et al., 2003; Ferreira et al., 2012), prompting a focus on reward region responsivity.

Few prospective studies have investigated neural vulnerability factors that predict future weight gain, which is necessary to ensure that they are precursors rather than consequences of overeating. Elevated responsivity of reward regions (striatum, amygdala, OFC) to palatable food images (Demos et al., 2012), 
palatable food commercials (Yokum et al., 2014), cues that predict palatable food image presentation (Yokum et al., 2011), and palatable food receipt (Geha et al., 2013) predicted future weight gain, converging with evidence from this same sample that healthy-weight youth at high versus low risk for future weight gain by virtue of parental obesity showed greater reward region response to high-calorie food receipt (Stice et al., 2011). Results are consistent with the reward surfeit model, which posits that greater reward region responsivity to food intake increases risk for overeating (Stice et al., 2008b) and the incentive sensitization model, which posits that cues that are repeatedly associated with palatable food intake come to activate reward regions and that this elevated reward region responsivity to food cues prompts overeating (Berridge et al., 2010).

There is evidence that the TaqIA polymorphism (rs1800497) may moderate these predictive effects: elevated dorsal striatum response to palatable food intake and images predicted future weight gain for adolescents with a genetic propensity for greater DA signaling because they possess the A2/A2 allele, but lower dorsal striatum response to these events predicted future weight gain for adolescents with a genetic propensity for lower DA signaling because they possess an A1 allele (Stice et al., 2008a, 2010). Individuals with the A2/A2 genotype have 30-40\% more DA D2 receptors and greater reward region responsivity than individuals with an A1/A1 or A1/A2 genotype (Bowirrat and Oscar-Berman, 2005). These data appear to provide support for the reward surfeit theory of obesity for individuals with a genetic propensity for greater DA signaling, and for the reward deficit theory of obesity, which posits that individuals with less responsive reward circuitry may overeat to compensate for a reward deficit (Volkow et al., 2008), for individuals with a genetic propensity for lower DA signaling.

The present study provided a more sensitive test of the relation of reward region response to receipt and anticipated receipt of palatable food and future weight gain over a longer period in a larger sample, and tested whether the predictive effects are moderated by the TaqIA allele. To rule out the possibility that a history of overeating contributed to any aberrant neural responsivity at baseline, we recruited a sample of healthy-weight adolescents, allowing the prediction of initial excessive weight gain. We predicted increases in percentage body fat, rather than body mass index scores, as the former provides a more direct measure of adipose tissue volume and is not influenced by variation in lean muscle mass. Another lacuna is that no study has tested whether the predictive effects of reward region responsivity is specific to food reward, or also emerges in response to alternative rewards. Thus, participants completed fMRI paradigms assessing responsivity to receipt and anticipated receipt of palatable food and monetary reward.

\section{Materials and Methods Participants}

A total of 162 adolescents [ 82 female, 80 male; mean age $=15.3 \pm 1.1$; mean body mass index $(\mathrm{BMI})=20.8 \pm 1.9 \mathrm{~kg} / \mathrm{m}^{2} ; 4 \%$ Hispanic, $1 \%$ Native American, $1 \%$ Asian/Pacific Islanders, 76\% European Americans, and $18 \%$ mixed racial heritage] were recruited in a US city via advertisements for a 3-year prospective study. Exclusion criteria were a BMI $<18$ or $>25$, current use of psychoactive medications or drugs more than weekly, pregnancy, head injury with a loss of consciousness, significant cognitive impairment, major medical problems, or current Axis I psychiatric disorder. Adolescents and parents provided written informed consent for this Institutional Review Board-approved project. Of the 162 enrolled, 7 participants provided only baseline data and thus were excluded from the present analyses and 2 participants' fMRI data were collected with an acquisition error resulting in altered deformation field output, rendering the data unusable with the methods described below. Findings from the resulting sample of 153 are reported.

\section{Measures}

fMRI paradigms. On the scan day, participants were asked to consume their regular meals, but to refrain from eating or drinking caffeinated beverages for $4-6 \mathrm{~h}$ preceding their scan. More than $85 \%$ of the scans occurred after school on weekdays (in the late afternoon), though some occurred in the late morning on no-school days.

The food reward fMRI paradigm (Fig. 1A) assessed response to receipt and anticipated receipt of a palatable chocolate milkshake. Stimuli were two images (glasses of milkshake and water) that signaled impending delivery of either $0.5 \mathrm{ml}$ of milkshake or tasteless solution (respectively). On $40 \%$ of the trials the taste was not delivered following the cue to allow investigation of neural response to anticipation of a taste that was not paired with receipt of the taste (unpaired trials). However, because no difference in response was observed between paired and unpaired milkshake and tasteless solution cues, paired and unpaired cues were combined for analyses to increase sensitivity. In total there were 30 repeats of both milkshake receipt and tasteless solution receipt, and 50 repeats of both the milkshake cue and the tasteless solution cue. Tastes were delivered using programmable syringe pumps. Syringes filled with milkshake and tasteless solution were connected via Tygon tubing to a manifold that fit into participants' mouths and delivered the taste to a consistent tongue segment. Participants were instructed when to swallow.

The monetary reward fMRI paradigm assessed activation in response to receipt and anticipated receipt of monetary reward (Fig. 1B). First a coin on the left side of the screen alternates between blinking heads $(\mathrm{H})$ and tails (T) 2-4 times for 300 ms per blink and then "lands" on either $\mathrm{H}$ or T. After $2 \mathrm{~s}$, a second coin in the middle of the screen blinks 4-6 times before landing on $\mathrm{H}$ or T. After $3 \mathrm{~s}$, a third coin blinks 8-10 times on the right side of the screen before landing on $\mathrm{H}$ or $\mathrm{T}$. After the presentation of the coins, a message appeared saying; "You win \$3" or "You don't win". There were 20 win events (HHH or TTT displays), 30 win anticipation events (HH or TT displays), and 30 reward-neutral events (when a single $\mathrm{H}$ or $\mathrm{T}$ was displayed, which conveyed no information about the probability of winning). All participants received $\$ 48$ once the scan was complete.

Activation in response to palatable food receipt was assessed by contrasting blood oxygen level-dependent (BOLD) signal during receipt of milkshake versus tasteless solution; activation in response to anticipated palatable food receipt was assessed by contrasting BOLD signal during the milkshake cue versus tasteless solution cue; activation in response to monetary reward was assessed by contrasting BOLD signal when participants saw the HHH or TTT displays versus the reward-neutral coin displays; activation in response to anticipated monetary reward was assessed by contrasting BOLD activation when participants saw a $\mathrm{HH}$ or a TT display versus the reward-neutral coin displays. These paradigms have been shown to result in greater activation of regions implicated in reward (orbitofrontal cortex, putamen, midbrain), as well as attention, oral somatosensory, and gustatory regions (Stice et al., 2012).

Food and monetary reinforcement. Participants completed the progressive reinforcement paradigm developed by Epstein et al. (2003), wherein they worked to earn points toward a snack food reward of their choice and monetary reward to provide behavioral data on sensitivity to food and monetary reward. Participants first performed a taste test of $1 \mathrm{~g}$ of each food (M\&Ms, Reese's, Kit Kat, Cheetos, Pringles, Skittles, Oreo cookies, Gingersnaps, Gummy Bears, and popcorn) and rated the pleasantness on cross-modal visual analog scales. They then selected the snack food they wanted to earn in the progressive reinforcement task. In the second phase, three boxes varying in shape and color were displayed on a computer screen (similar to a slot machine display). The boxes flipped, rotated, and changed color each time the participant pressed the mouse button. Points were earned each time the shapes matched in color and shape. The task started at a variable ratio $1 / 4$ schedule meaning that, on average, one point was awarded for four button presses. The progressive ratio schedule for the food item doubled (VR8, VR16, VR32, etc.) each time they earned five points. They were told that it would get progres- 

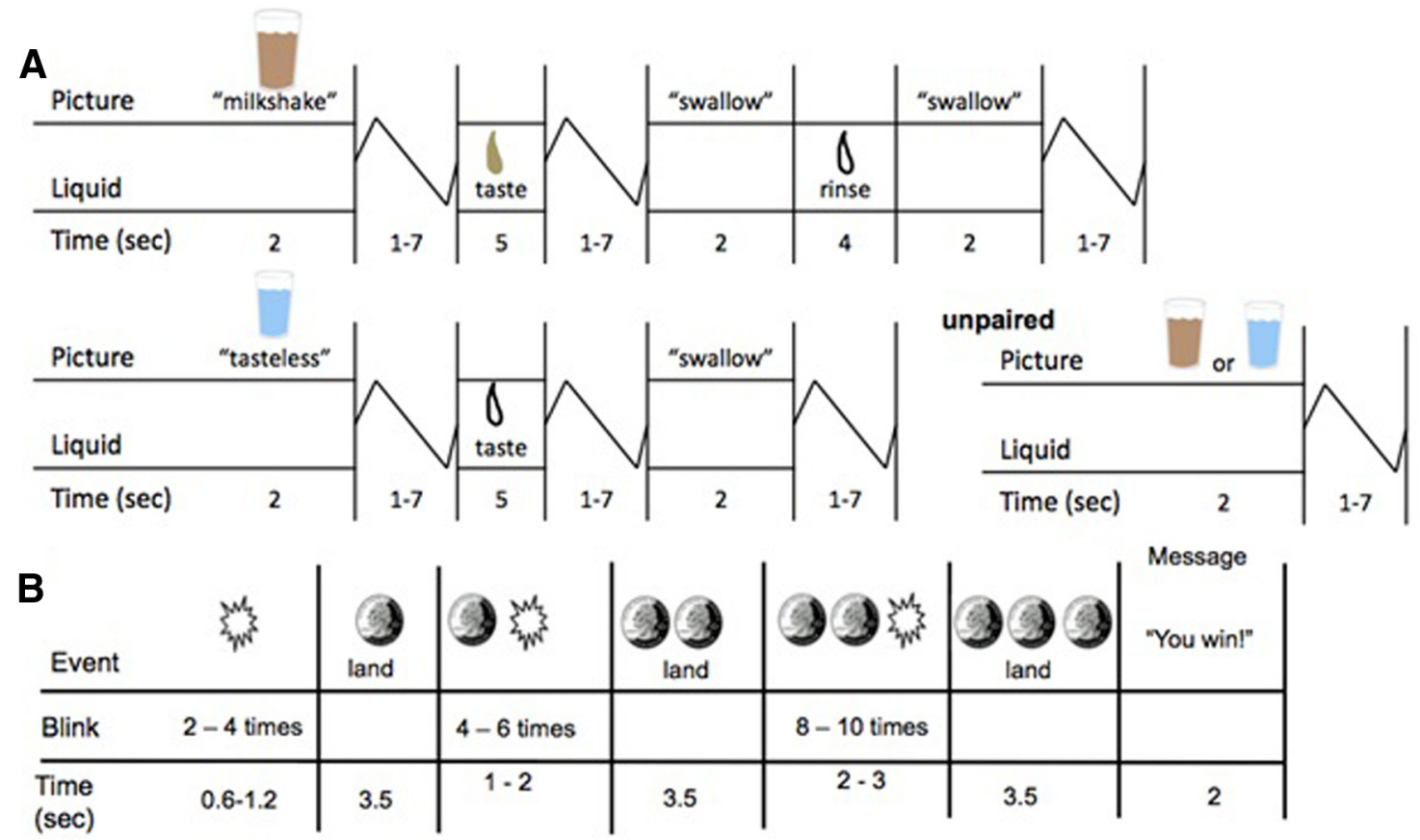

Figure 1. Example of timing and ordering of presentation of pictures and beverages during the food reward paradigm $(\boldsymbol{A})$ and of presentation of images and notification of monetary reward during the monetary reward paradigm $(\boldsymbol{B})$.

sively harder to earn points. The number of points earned for snacks was displayed at the top of the screen. Each 5 points earned was worth 1 standard serving of the food. Participants were told to play for as long as they liked. They then repeated this paradigm, but worked for $\$ 1$ monetary rewards, to provide a behavioral measure of sensitivity to an alternative reward. The breakpoint at which the participant stopped button pressing for food was used as the behavioral measure of food reward (i.e., how many button presses are made in total before the subject stops). A similar monetary reward breakpoint was calculated, as was the relative breakpoint for food versus money reward. The food reinforcement paradigm has shown 2-7 d test-retest reliability $(r=0.80$; Epstein et al., 2007). Participants who rate the snack food as more pleasant work longer for it, participants who work longer for the snack consume more food ad libitum, participants work longer for snack food when calorically deprived, and obese versus lean participants work longer for snack food (Epstein et al., 2003, 2007; Goldfield and Legg, 2006).

Genotyping. Participants provided saliva from which epithelial cells were collected using a commercial product, Oragene (DNA Genotek). DNA was extracted from the samples using standard salting-out and solvent precipitation methods, yielding an average of $45 \mu \mathrm{g}$ of DNA. The TaqIA (rs1800497) assay was performed using a fluorogenic $5^{\prime}$-nuclease (Taqman, Applied Biosystems) method (Haberstick and Smolen, 2004) on an ABI Prism 7000 Sequence Detection System via the allelic discrimination mode (Livak, 1999). Reactions containing $20 \mathrm{ng}$ of DNA were performed in $10 \mu \mathrm{l}$ reactions with TaqMan Universal PCR Master Mix using the standard cycling conditions. Sequences of the TaqIA primers and probes are as follows: forward primer: $5^{\prime}$-GTGCAGCTCACTCCATCCT-3'; reverse primer: 5'-GCAACACAGCCATCCTCAAAG-3'; A1 probe: $5^{\prime}$-VIC-CCTGCCTTGACCAGC-MGB-3'; A2 probe: $5^{\prime}$ FAM-CTGCCTCGACCAGC-MGB-3'. Each 96 well plate included nontemplate and DNA standards of known genotype. Three genotype groups were defined for TaqIA: A1 homozygotes $(n=4)$, A1/A2 heterozygotes $(n=55)$, and A2 homozygotes $(n=94)$. The TaqIA site resides in exon 8 of the ANKK1 gene on the opposite strand. This SNP results in a glutamate-to-lysine (E713K) substitution within the 11th ankyrin repeat of ANKK1 (Neville et al., 2004).

Percentage body fat. Air displacement plethysmography was used to assess percentage body fat of participants at baseline and at 1-, 2-, and 3 -year follow-up with the Bod Pod S/T using recommended procedures and age/sex-appropriate equations (Lohman, 1989). Body density was calculated as body mass (assessed by direct weighing) divided by body volume. Body fat percentage estimates show test-retest reliability $(r=$ 0.92-0.99) and correlate with dual X-ray absorptiometry and hydrostatic weighing estimates ( $r=0.98-0.99$; Fields et al., 2002). Analyses predicted within-subject change in body fat percentage rather than BMI because the latter scores are biased high for participants with elevated lean muscle mass. We did not use age-adjusted BMI or body fat percentage scores because methodologists have argued that it is better to model within-subject change in raw scores, rather than those adjusted to remove variation due to aging when predicting change over development (Berkey and Colditz, 2007).

\section{Analytic plan}

Body fat data from baseline, and 1-, 2-, and 3-year follow-ups were used in random intercept, mixed effects growth curve analyses (SAS Inc. version 9.3) to model body fat change, which offer a sensitive technique for modeling change in continuous variables and use maximum likelihood estimation to accommodate missing data (Singer, 1998). Following the method of Singer and Willet (2003), we: (1) examined empirical growth plots; (2) fit an unconditional means model; (3) fit an unconditional linear growth model; and (4) fit unconditional nonlinear models. We compared the latter two models using the Akaike information criterion (AIC) to determine whether linear or higher-order polynomial models fit the data better. AIC is a measure of goodness of fit relative to model complexity (Burnham and Anderson, 2002). Compared with higherlevel polynomial models, linear growth models consistently showed a better fit per AIC values, suggesting that linear terms optimally captured change in body fat over time.

Scanning was performed using a Siemens Allegra 3 Tesla head-only MRI scanner. A birdcage coil acquired data from the entire brain. A thermo foam vacuum pillow and additional padding restricted head motion. Functional scans used a $\mathrm{T} 2^{\star}$-weighted gradient single-shot echo planar imaging $(\mathrm{EPI})$ sequence $(\mathrm{TE}=30 \mathrm{~ms}, \mathrm{TR}=2000 \mathrm{~ms}$, flip angle $=$ $\left.80^{\circ}\right)$ with an in-plane resolution of $3.0 \times 3.0 \mathrm{~mm}^{2}(64 \times 64$ matrix; $192 \times 192 \mathrm{~mm}^{2}$ field of view). To cover the whole brain, 32 slices, $4 \mathrm{~mm}$ (interleaved acquisition, no skip), were acquired along the AC-PC transverse, oblique plane as determined by the midsagittal section. Structural scans were collected using an inversion recovery T1-weighted sequence 
(MP-RAGE) in the same orientation as the functional sequences to provide detailed anatomic images aligned to the functional scans. Highresolution structural MRI sequences $\left(\mathrm{FOV}=256 \times 256 \mathrm{~mm}^{2}, 256 \times 256\right.$ matrix, thickness $=1.0 \mathrm{~mm}$, slice number $\approx 160$ ) were acquired.

Neuroimaging data were preprocessed and analyzed primarily using SPM12 (Functional Imaging Laboratory, University College London) in Matlab (MathWorks) for Mac OSX. Before preprocessing, all images were manually realigned to the AC-PC line in SPM and skull-stripped using the Brain Extraction Tool in FSL (FMRIB Analysis Group, Oxford, UK). During preprocessing in SPM, anatomical data were segmented and normalized using DARTEL, resulting in a sample-specific template and individual-level deformation fields for application to the normalization step during functional data preprocessing. Functional data were as follows: (1) slice timing corrected, as these methods can successfully compensate for the temporal offset between slice acquisition and increase the robustness of the data analysis (Sladky et al., 2011); (2) adjusted for variation in magnetic field distortion using field maps (Poldrack et al., 2011); (3) realigned to the mean functional from that run and coregistered with the anatomical; and (4) normalized to Montreal Neurological Institute (MNI) space using the DARTEL template and deformation fields output, which allows more precise alignment (Klein et al., 2009). Last, functional data were smoothed to $6 \mathrm{~mm}$ Gaussian FWHM. Functional data were then assessed to detect spikes in global mean response and motion outliers in the functional data using the Artifact Detection Toolbox (ART; Gabrieli Laboratory, McGovern Institute for Brain Research, Cambridge MA). Motion parameters were included as regressors in the design matrix at individual-level analysis. Additionally, image volumes where the $z$-normalized global brain activation exceeded 3 SDs from the mean of the run or showed $>1 \mathrm{~mm}$ of composite (linear plus rotational) movement were flagged as outliers and deweighted during individual-level model estimation.

At the individual-level, T-maps were constructed for comparison of activation within each participant for the four contrasts on the individual level (e.g., milkshake receipt $>$ tasteless solution receipt). These individual contrasts were entered into a second-level regression model with body fat slopes and intercepts that were calculated using the aforementioned random intercept, mixed effects growth curve analyses. Wholebrain analyses were conducted after the binarized DARTEL-derived sample-specific gray matter mask was applied. An overall significance level of $p<0.05$ corrected for multiple comparisons across the gray matter-masked whole brain was calculated. This calculation was accomplished by: (1) estimating the inherent smoothness of the masked functional data with the 3dFWHMx module in AFNI (Cox, 1996); and (2) performing 10,000 Monte Carlo simulations of random noise at $3 \mathrm{~mm}^{3}$ through the masked data using the 3DClustSim module of AFNI (Forman et al., 1995). Simulation results indicated activity surviving a threshold of $p<0.005$, with a cluster $(k) \geq 26$ being statistically significant corrected for multiple comparisons. Split-half replication was used to test the stability of the main effects predictive analyses. These were accomplished using block randomization of male and female participants into two equal groups. Main effect predictive analyses were performed on both groups separately and results were compared with one another. Results from analyses of main effect predictive analyses were tested using random split-half replication tests thresholded at $p<0.005 ; k \geq 10$. Main effect predictive results presented were present in the split-half tests to reduce the possibility that models were overfit to the data (Whelan and Garavan, 2014). For a priori tests seeking to replicate the previous interaction involving the TaqIA polymorphism, regions of interest (ROIs) were used. For these analyses, $10 \mathrm{~mm}$ spheres were drawn centering on the peak voxel from the previously published papers (Stice et al., $2008 \mathrm{a}, \mathrm{b})$. For ROIs, peak activity with $p$-values $<0.05$ corrected using voxel-level familywise error rate (pFWE) over the $10 \mathrm{~mm}$ sphere were considered significant.

Data were inspected to ensure that influential outliers did not drive significant effects. Baseline age, self-reported Tanner stage, sex, hunger, milkshake pleasantness ratings, and menstrual phase did not significantly predict body fat change and were therefore not potential confounds, as a variable must explain variance in the outcome to confound the relation between the independent and dependent variable (Cohen et al., 2003).
Table 1. Subject characteristics at each annual assessment for the total sample as well as for males and females separately

\begin{tabular}{lrrr}
\hline & \multicolumn{1}{l}{ Total $N$} & \multicolumn{1}{l}{ Male } \\
\hline Baseline & & & \\
$\quad$ Age & $15.3 \pm 1.1$ & $15.4 \pm 1.2$ & $15.2 \pm 1.0$ \\
BMI \pm SD & $20.8 \pm 2.0$ & $20.7 \pm 2.0$ & $20.9 \pm 1.9$ \\
BMI z-score \pm SD & $0.07 \pm 0.7$ & $-0.00 \pm 0.7$ & $0.15 \pm 0.5$ \\
$\quad$ Body fat \% \pm SD & $18.6 \pm 7.7$ & $12.8 \pm 5.4$ & $23.9 \pm 5.3$ \\
1-year follow-up & & & \\
Age & $16.3 \pm 1.1$ & $16.3 \pm 1.2$ & $16.2 \pm 1.0$ \\
BMI \pm SD & $21.5 \pm 2.3$ & $21.7 \pm 2.6$ & $21.4 \pm 2.0$ \\
BMI z-score \pm SD & $0.09 \pm 0.8$ & $0.06 \pm 0.9$ & $0.11 \pm 0.6$ \\
Body fat \% \pm SD & $18.9 \pm 7.9$ & $13.0 \pm 5.3$ & $24.1 \pm 5.8$ \\
2-year follow-up & & & \\
Age & $17.3 \pm 1.1$ & $17.3 \pm 1.1$ & $17.2 \pm 1.0$ \\
BMI \pm SD & $21.9 \pm 2.4$ & $22.1 \pm 2.6$ & $21.6 \pm 2.2$ \\
BMI z-score \pm SD & $0.04 \pm 0.8$ & $0.01 \pm 0.9$ & $0.07 \pm 0.7$ \\
Body fat \% \pm SD & $19.7 \pm 8.3$ & $13.7 \pm 6.4$ & $24.8 \pm 6.0$ \\
3-year follow-up & & & \\
Age & $18.2 \pm 1.1$ & $18.2 \pm 1.1$ & $18.2 \pm 1.0$ \\
BMI \pm SD & $22.4 \pm 3.0$ & $22.6 \pm 3.0$ & $22.3 \pm 2.9$ \\
BMI Z-score \pm SD & $-0.01 \pm 0.9$ & $-0.0 \pm 1.0$ & $-0.02 \pm 0.8$ \\
Body fat \% \pm SD & $20.4 \pm 9.3$ & $13.9 \pm 7.0$ & $26.2 \pm 7.2$ \\
\hline
\end{tabular}

Consistent with this logic, we confirmed that all predictive effects reported below remained significant when we controlled for these potential confounds. Effect sizes $(r)$ were derived from the $Z$-values $(Z / \sqrt{ } N)$.

\section{Results}

\section{Preliminary analyses}

Table 1 shows the subject characteristics at each annual assessment for the total sample as well as for males and females separately. The average BMI $\left(\mathrm{kg} / \mathrm{m}^{2}\right)$ was $20.8 \pm 2.0$ at baseline and $22.4 \pm 3.0$ at 3 -year follow-up. Average change in BMI over 3 -year follow-up was $1.6 \pm 2.2$ (range -2.6 to 14.3 ). The average body fat percentage was $18.6 \pm 7.7$ at baseline and $20.4 \pm 9.3$ at 3 -year follow-up. Average change in body fat percentage over the 3 -year follow-up was $1.7 \pm 5.7$ (range -12.3 to 22.3 ). Based on percentage body fat scores, 25 participants showed initial onset of overweight (16\%) and 18 showed initial onset of obesity (12\%) over follow-up. Figure 2 graphs body fat percentage at baseline versus 3-year follow-up, illustrating the individual differences in body fat gain over follow-up.

\section{Relation of neural responsivity to body fat change over 3-year follow-up}

Elevated BOLD response to the contrast of the milkshake cue $>$ tasteless solution cue, which putatively reflects greater anticipatory palatable food reward, in the OFC and frontal pole (Fig. 3; Table 2) predicted body fat gain over the 3-year follow-up. Elevated BOLD response to the contrast of milkshake receipt $>$ tasteless solution receipt in the precuneus likewise predicted body fat gain over 3-year follow-up (Fig. 4A; Table 2). Lower BOLD activity to the milkshake cue $>$ tasteless solution cue contrast in the bilateral superior visual cortex, lingual gyrus, and ventromedial prefrontal cortex (vmPFC) also predicted body fat gain over follow-up (Fig. 4B; Table 2). Further, lower BOLD response to the palatable food receipt contrast in the anterior superior parietal lobe predicted body fat gain over follow-up (Table 2). All results remained significant when controlling for time of day at which the scan was performed. Sex did not significantly moderate any of these predictive effects.

Individual differences in BOLD response to the contrast of the display signaling monetary reward $>$ the reward-neutral coin 


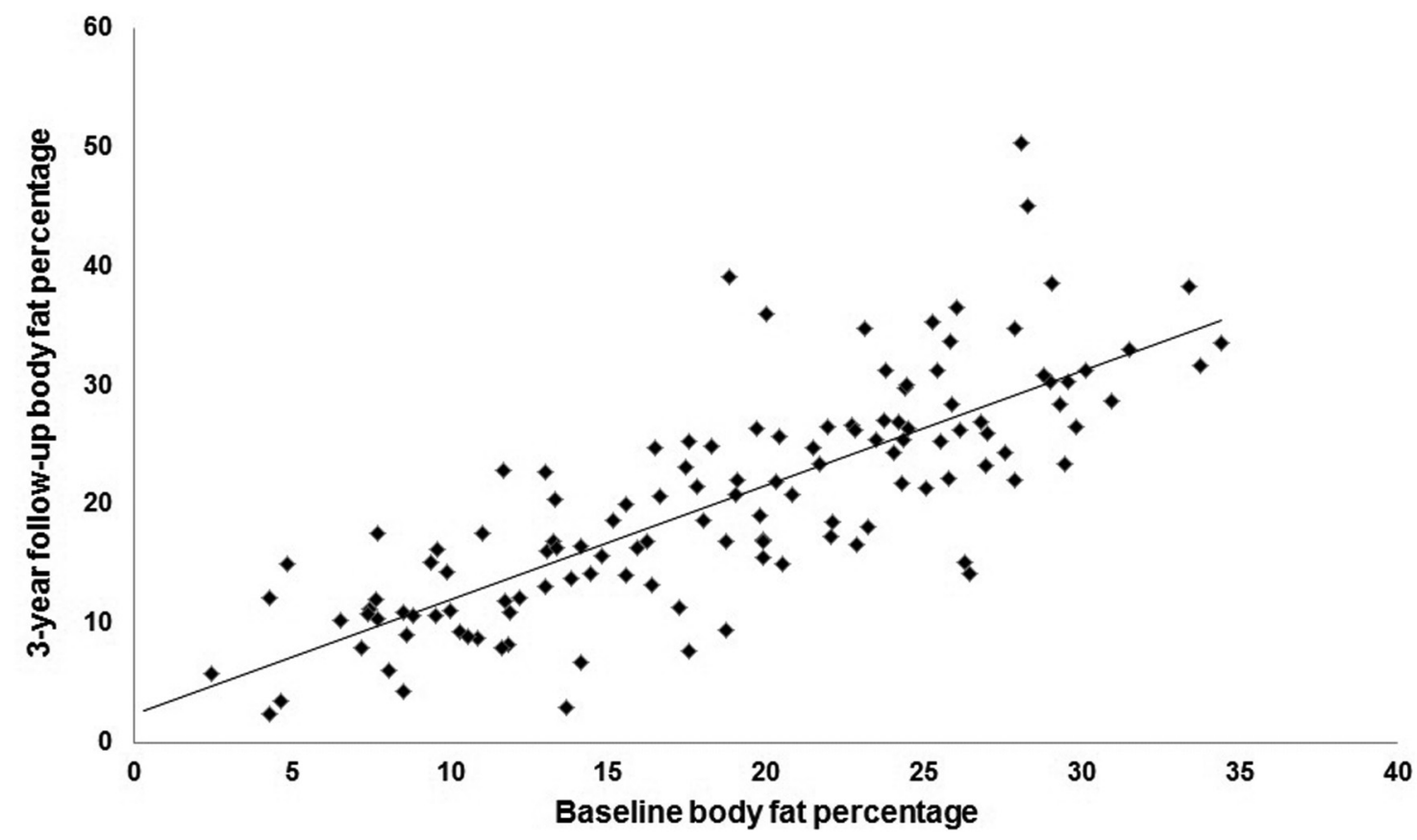

Figure 2. Correlation between baseline body fat percentage and 3-year follow-up body fat percentage.

display, and in response to the contrast of the display signaling a potential monetary reward $>$ the reward-neutral coin display did not significantly predict future body fat gain.

\section{Moderators of the relation of neural responsivity to body fat change over 3-year follow-up}

The interaction between BOLD response in the caudate ROI to milkshake receipt $>$ tasteless solution receipt and the TaqIA polymorphism was a marginal trend (pFWE $=0.06)$ (Table 2). Simple slopes analyses indicated that greater BOLD response in the caudate during milkshake receipt $>$ tasteless solution receipt contrast significantly predicted body fat gain for participants with an A2/A2 allele ( $r=$ $0.25 ; p=0.02$ ), whereas lower BOLD response showed a marginal correlation with future body fat gain for participants with one or more A1 alleles ( $r=$ $-0.24 ; p=0.06$; Fig. $4 \mathrm{C}$ ).

Given that in the process of writing an earlier report (Stice et al., 2011) we discovered that adolescents with versus without a parental history of obesity had nearly a twofold greater prevalence of the TaqIA A1 allele (43\% versus 22\%, Mantel Haenszel odds ratio $=2.7 ; 95 \% \mathrm{CI}: 1.1-6.1, p=0.03)$, we tested whether parental obesity moderated the relation of caudate response to milkshake and future body fat gain. This seemed important because parental obesity is one of the most robust risk factors for obesity (Magarey et al., 2003). In the present sample of 153, 118 were children of two obese or overweight (BMI $\geq 27$ ) parents and 35 were children of two lean parents (by design we over-recruited the former and excluded adolescents with one

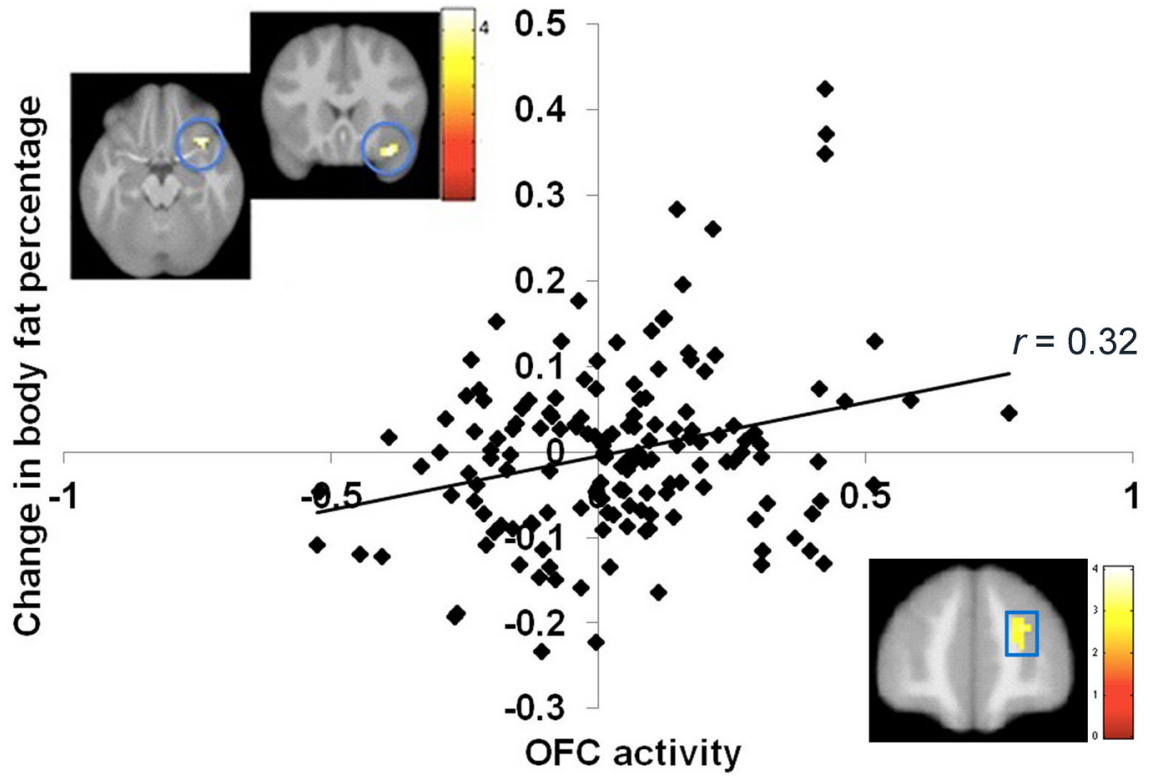

Figure 3. Greater BOLD response and parameter estimates from the orbitofrontal cortex (circles; MNI coordinates: 33, 18, - 21, $Z=3.9$ ) and greater BOLD response in a region in the frontal pole (square; $\mathrm{MNI}$ coordinates: $24,51,21, Z=3.3$ ) in response to the anticipatory milkshake cue-predicted future body fat gain over 3-year follow-up.

lean and one overweight/obese parent). The interaction between BOLD response in the caudate ROI to milkshake receipt $>$ tasteless solution receipt and parental obesity was significant (Table 3). Similar to the interaction observed with TaqIA allele status, simple slope analyses revealed that greater BOLD response in the caudate during milkshake receipt $>$ tasteless solution receipt contrast significantly predicted future body fat gain for participants without parental obesity $(r=0.54, p=0.001)$, but lower BOLD response correlated with body fat gain for participants with parental obesity, though this relation did not reach statistical significance $(r=$ $-0.15, p=0.12$; Fig. $4 \mathrm{D})$. 


\begin{tabular}{|c|c|c|c|c|}
\hline & $k$ & $Z$ value & $\begin{array}{l}\text { MNI } \\
\text { coordinates }\end{array}$ & $r$ \\
\hline \multicolumn{5}{|l|}{$\begin{array}{l}\text { Milkshake receipt }>\text { tasteless solution receipt } \\
\text { Positive relation weight change }\end{array}$} \\
\hline Precuneus & 85 & $\begin{array}{l}3.4 \\
2.9\end{array}$ & $\begin{array}{l}0,-60,30 \\
6,-46,36\end{array}$ & $\begin{array}{l}0.28 \\
0.24\end{array}$ \\
\hline \multicolumn{5}{|l|}{ Negative relation weight change } \\
\hline Anterior superior parietal lobe & 53 & $\begin{array}{l}-3.8 \\
-3.2 \\
-2.8\end{array}$ & $\begin{array}{l}30,-45,51 \\
30,-36,48 \\
33,-33,39\end{array}$ & $\begin{array}{l}-0.31 \\
-0.26 \\
-0.23\end{array}$ \\
\hline \multicolumn{5}{|l|}{$\begin{array}{l}\text { Milkshake cue }>\text { tasteless solution cue } \\
\text { Positive relation weight change }\end{array}$} \\
\hline Frontal pole & $\begin{array}{l}15 \\
26\end{array}$ & $\begin{array}{l}3.9 \\
3.3 \\
2.7\end{array}$ & $\begin{array}{l}33,18,-21 \\
24,51,21 \\
12,57,18\end{array}$ & $\begin{array}{l}0.32 \\
0.27 \\
0.22\end{array}$ \\
\hline \multicolumn{5}{|l|}{ Negative relation weight change } \\
\hline Superior visual cortex/Cuneus (right) & 81 & $\begin{array}{l}-4.0 \\
-3.0\end{array}$ & $\begin{array}{l}24,-66,27 \\
18,-75,33\end{array}$ & $\begin{array}{l}-0.33 \\
-0.24\end{array}$ \\
\hline Lingual gyrus & 19 & -3.5 & $18,-42,-9$ & -0.28 \\
\hline $\begin{array}{l}\text { Superior visual cortex/Cuneus (left) } \\
\text { vmPFC }\end{array}$ & $\begin{array}{l}28 \\
14\end{array}$ & $\begin{array}{l}-3.3 \\
-3.2\end{array}$ & $\begin{array}{l}-15,-75,18 \\
-9,39,0\end{array}$ & $\begin{array}{l}-0.27 \\
-0.26\end{array}$ \\
\hline
\end{tabular}

For all contrasts, activated regions, number of contiguous voxels (k), Z-values, and coordinates within the MNI coordinate system are displayed.

\section{Reinforcement as a predictor of weight gain}

Using the growth curve modeling techniques described above, monetary reinforcement predicted increases in body fat $(r=$ $0.16 ; p=0.048)$. However, food reinforcement $(r=0.01 ; p=$ 0.87 ) and relative reinforcement (food reinforcement/monetary reinforcement; $r=0.00 ; p=0.93$ ) did not predict future body fat gain.

\section{Behavioral correlates of BOLD response to milkshake receipt and anticipated receipt}

Precuneus response from the milkshake receipt $>$ tasteless solution receipt contrast was positively related to monetary reinforcement $(r=0.19 ; p=0.02)$, indicating that those who worked more for monetary reward showed greater precuneus response. However, the precuneus and anterior superior parietal lobe peaks from the milkshake receipt $>$ tasteless solution receipt contrast (Table 1) were not significantly associated with ratings of hunger $(r$ values $=-0.05,-0.01)$, milkshake pleasantness $(r$ values $=$ $0.05,-0.06)$, food reinforcement $(r$ values $=0.09,0.10)$, or relative reinforcement for food versus monetary reward $(r$ values $=$ $-0.05,0.11)$. No significant relations were observed between activity peaks during milkshake cue $>$ tasteless solution cue and the aforementioned behavioral measures ( $r$ values $=-0.13$ to 0.10 ).

\section{Discussion}

Whole-brain analyses indicated that greater right lateral OFC and frontal pole response to cues signaling impending milkshake receipt predicted body fat gain over 3-year follow-up, converging with evidence that greater lateral OFC response to cues signaling impending palatable food image presentation predicted future weight gain (Yokum et al., 2011). Because the lateral OFC plays a role in learning reward-specific associations (Noonan et al., 2012), this finding implies that youth who show elevated activation in this reward-learning region to palatable food cues are at risk for overconsumption of calories relative to caloric expenditure, which is necessary for increases in adipose stores (Hall et al., 2012). As activity in the right frontal pole has been implicated in working memory (Soto et al., 2007), that peak may imply that individuals better able to represent and recall the hedonic value from palatable foods are at risk for overeating. Collectively, data appear consistent with the incentive sensitization theory of obesity (Berridge et al., 2010), as they imply that youth who show greater reward region responsivity to food cues and who may better recall the hedonic pleasure for palatable food are at risk for overeating.

We found a trend-level interaction between caudate response to palatable food receipt and TaqIA allele status in the prediction of body fat gain, replicating an earlier finding (Stice et al., 2008a). Elevated caudate response to milkshake receipt predicted body fat gain for youth with a genetic propensity for greater DA signaling due to having a TaqIA A2/A2 allele, but lower caudate response predicted body fat gain for youth with a genetic propensity for less DA signaling due to having a TaqIA A1 allele. Although the effect size was smaller in this sample, the fact that a similar effect emerged in a lean sample that included both sexes and from a somewhat different paradigm implies this interaction is robust. The effect might have been larger in the earlier sample because in contrast to the present sample, it included overweight and obese youth, implying that the effect may be stronger among individuals who have already reached an obese state or who are at higher risk for obesity (as indexed by the fact that many had already expressed this risk). Interestingly, parental obesity correlated with TaqIA A1 allele status and similarly moderated the predictive effects of caudate response to milkshake receipt. The former interaction suggests the possibility of qualitatively distinct reward surfeit (Stice et al., 2008b) and reward deficit (Volkow et al., 2008) pathways to obesity, implying that too little or too much DA signaling and reward region responsivity may both increase risk for overeating. These results, taken in conjunction with evidence that individuals who show a greater propensity for cue-reward learning exhibit elevated future weight gain (Burger and Stice, 2014), suggest that researchers should investigate whether individuals who show greater reward prediction signals and reward prediction error signals in response to food cues that are mediated by dopamine signaling in reward circuitry (Schultz, 2010) exhibit greater future weight gain. We were unable to model prediction error signaling with the current fMRI paradigm because the jitter between the milkshake cue and milkshake receipt made it impossible for the participant to determine when a reward prediction error occurred.

Greater precuneus response to milkshake receipt predicted future body fat gain. Precuneus activity occurs in response to salient stimuli (Tang et al., 2012), has been associated with preferences for palatable food (Small et al., 2001), and is functionally connected to reward regions (e.g., striatum and midbrain; Cavanna and Trible, 2006). This finding may therefore suggest that participants who found the milkshake more salient are at risk for overeating. In contrast, anterior superior parietal lobe response to milkshake receipt predicted less body fat gain. The anterior superior parietal lobe is involved in the integration of neural signals from different sensory modalities (Sereno and Huang, 2014) and is activated during tactile processing (Swisher et al., 2007). Thus, results may suggest that those who show less attention or sensitivity to the taste properties of the milkshake (e.g., texture) are at risk for overeating.

Lower response in visual processing regions (cuneus, lingual gyrus) and the left vmPFC to anticipatory milkshake receipt predicted future body fat gain. Activations in these regions are positively associated with visual attention and reward processing (Due et al., 2002; Hare et al., 2009). Lingual gyrus response to 

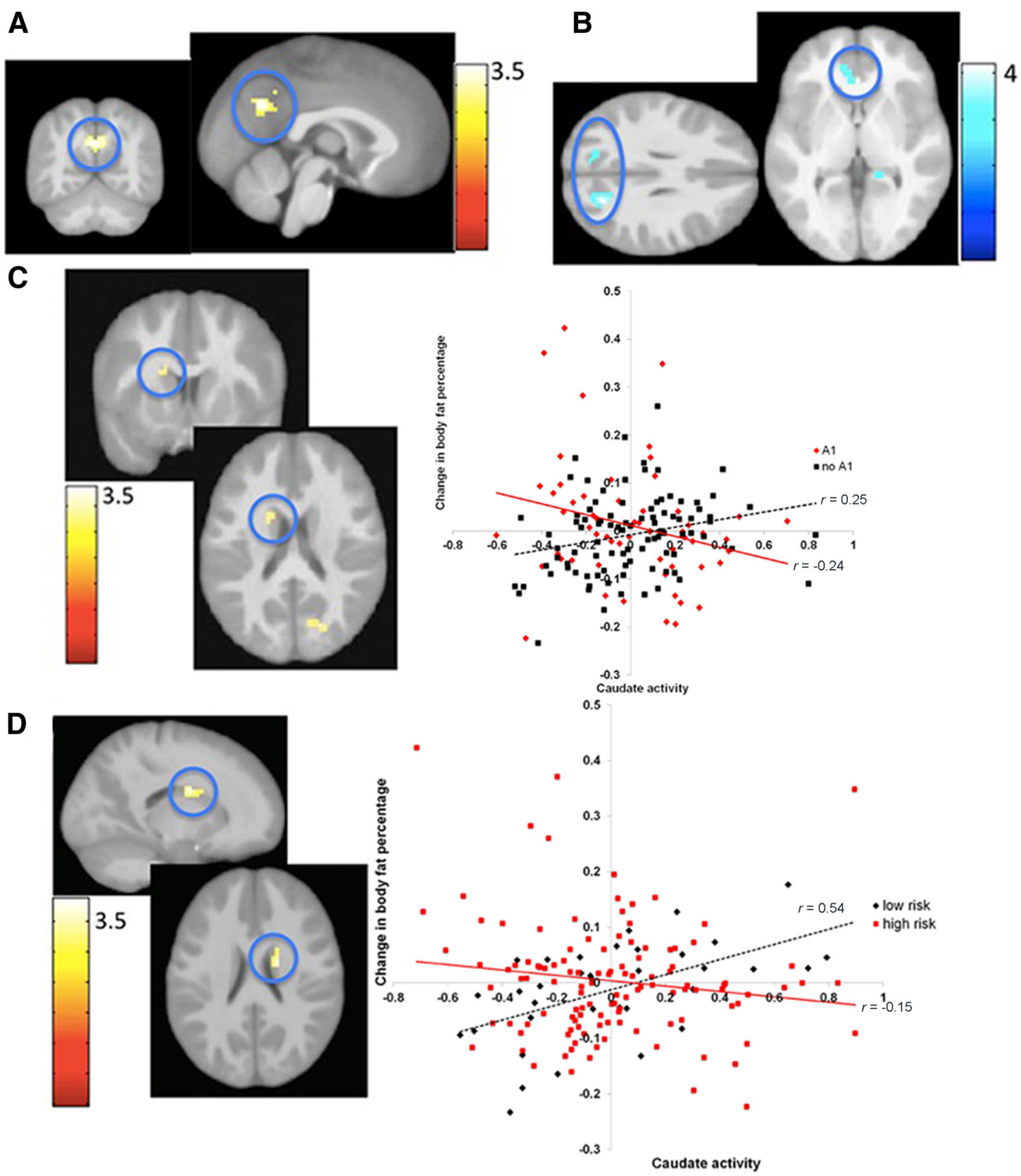

Figure 4. $A$, Greater BOLD response in the precuneus (MNI coordinates: $0,-60,30, Z=3.4, r=0.28$ ) in response to milkshake receipt predicted future body fat gain over 3-year follow-up. $B$, Lower BOLD response in the visual cortex, lingual gyrus and vmPFC ( $r$ values -0.40 to -0.32 ) predicted future body fat gain over 3-year follow-up. $C$, Greater BOLD response in the caudate during milkshake receipt predicted body fat gain for participants without an A1 allele, but lower caudate response predicted future body fat change for participants with the A1 allele (MNI coordinates: $-18,6,21, Z=3.0, r=0.24)$. D, Greater BOLD response in the caudate during milkshake receipt predicted future body fat gain for participants without parental obesity, but lower caudate response predicted body fat gain for participants with parental obesity (MNI coordinates: $18,-3,24, Z=3.8, r=0.31$ ).

Table 3. Interaction of BOLD response to milkshake receipt $>$ tasteless solution receipt and TaqIA allele status and parental obesity in the prediction of future body fat gain

$k$ Zvalue MNI coordinates $r \quad$ pFWE

Interaction between TaqIA A1 status and BOLD activation in response to milkshake receipt $>$ tasteless solution receipt in the prediction of

future body fat gain

Caudate

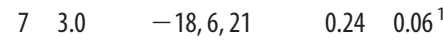

Interaction between obesity risk status and BOLD activation in response to milkshake receipt $>$ tasteless solution receipt in the prediction of

future body fat gain 
anticipatory milkshake receipt correlated positively with caloric intake (Burger and Stice, 2013). Obese versus lean individuals show greater response in visual processing regions and vmPFC to food images (Martin et al., 2010). Yet, another study found that obese versus lean adolescents showed less vmPFC response to palatable food commercials (Gearhardt et al., 2014). Activity in visual processing regions and vmPFC initially has been found to increase due to allocation of attention to novel events, but decreases over time due to a learning-related transition (Goldstein et al., 2007; Mundy et al., 2014). Weight gainers may have shown greater neural learning or habituation responses to the palatable food cues, perhaps due to a stronger food reward-cue learning propensity or prior experience with palatable food, consistent with the positive relation between lateral OFC response to anticipatory milkshake receipt and body fat gain. Alternatively, the negative associations of activity in the cuneus, lingual gyrus, and vmPFC with body fat gain might reflect a reduced effort or increased automaticity of attention to the palatable food cues in body fat gainers. The fact that no decisional processes were required by participants to consume the food as a function of the cues might have decreased the salience of the food cues. Perhaps in this scenario, those who are more likely to gain body fat show less visual region recruitment as they are more focused on palatable food receipt. Although speculative, this interpretation is consistent with the positive relation between precuneus activity and body fat gain.

Reward region response to receipt and anticipated receipt of monetary reward did not predict body fat gain, suggesting that overeating is rooted in elevated responsivity of reward regions to food, rather than a broader array of reinforcers. However, because food is a primary reinforcer and money a secondary reinforcer, it is possible that it is elevated reward region responsivity to primary reinforcers that increases overeating risk. Yet, monetary reward breakpoint predicted body fat gain, suggesting that general elevated reward sensitivity may increase overeating risk. Another difference between the two paradigms is that participants received the palatable food during the scans, but not the monetary reward. Thus, it is possible that the predictive effects emerged for the food but not the monetary reward paradigm because the former provided a more sensitive measure of reward region responsivity. However, greater dorsal striatum response to monetary reward receipt in this fMRI paradigm did predict future substance use onset (Stice et al., 2013), suggesting this paradigm has predictive validity.

Results represent a novel contribution in that no large study has investigated whether neural response to receipt and anticipated receipt of palatable food and monetary reward predicts future weight gain over such a long follow-up in such a large sample. The fact that all adolescents had a healthy weight at baseline reduces the possibility that previous overeating contributed to aberrant neural responsivity at baseline that predicted future weight gain. It also appears to be the first report in this research area to use split-half replication. Nonetheless, this study had limitations. First, we only examined response to a single high-calorie palatable food; it is unclear whether results will generalize to receipt of other foods. Second, although the follow-up period is longer than that used in any prior prospective study on neural response to food stimuli, many of the participants who did not gain weight over follow-up may do so in the future; such right censoring would reduce sensitivity. Third, our ability to predict body fat gain might have been limited by the fact that participants only completed fMRI scans at baseline. Future researchers should conduct annual fMRI scans over follow-up in concert with annual measures of body fat. Fourth, although we focused on changes in objectively measured body fat as our outcome because people under-report caloric intake and this under-reporting is greater for heavier individuals according to objective biological estimates of total caloric intake (Bandini et al., 1990), we could not confirm that increases in body fat were due to overconsumptions of calories relative to caloric expenditure.

In sum, elevated OFC response to cues signaling impending milkshake receipt predicted body fat gain, converging with previous findings and providing support for the incentive sensitization theory of obesity. Further, an interaction indicated that elevated caudate response to milkshake receipt predicted future body fat gain for youth with a genetic propensity for greater DA signaling, but lower caudate response predicted body fat gain for youth with a genetic propensity for less DA signaling, replicating earlier findings. The interactive results imply that too much or too little DA signaling capacity and reward region responsivity may increase risk for overeating, suggesting the possibility of qualitatively distinct reward surfeit and reward deficit pathways to obesity.

\section{References}

Bandini L, Schoeller D, Cyr H, Dietz W (1990) Validity of reported energy intake in obese and non-obese adolescents. J Clin Nutr 52:421-425. Medline

Berkey CS, Colditz GA (2007) Adiposity in adolescents: change in actual BMI works better than change in BMI $z$ score for longitudinal studies. Ann Epidemiol 17:44-50. CrossRef Medline

Berridge KC, Ho CY, Richard JM, DiFeliceantonio AG (2010) The tempted brain eats: pleasure and desire circuits in obesity and eating disorders. Brain Res 1350:43-64. CrossRef Medline

Bowirrat A, Oscar-Berman M (2005) Relationship between dopaminergic neurotransmission, alcoholism, and Reward Deficiency syndrome. Am J Med Genet B Neuropsychiatr Genet 132B:29-37. Medline

Burger KS, Stice E (2013) Elevated energy intake is correlated with hyperresponsivity in attentional, gustatory, and reward brain regions while anticipating palatable food receipt. Am J Clin Nutr 97:1188-1194. CrossRef Medline

Burger KS, Stice E (2014) Greater striatopallidal adaptive coding during cue-reward learning and food reward habituation predict future weight gain. Neuroimage 99:122-128. CrossRef Medline

Burnham KP, Anderson, D (2002) Model selection and multi-model inference: a practical information-theoretic approach. New York: Springer.

Cavanna AE, Trimble MR (2006) The precuneus: a review of its functional anatomy and behavioural correlates. Brain 129:564-583. CrossRef Medline

Cohen J, Cohen P, West S, Aiken L (2003) Applied multiple regression/ correlation analysis for the behavioral sciences, Ed 3. Mahwah, NJ: Lawrence Erlbaum Associates.

Cox RW (1996) AFNI: software for analysis and visualization of functional magnetic resonance neuroimages. Comput Biomed Res 29:162-173. CrossRef Medline

Demos KE, Heatherton TF, Kelley WM (2012) Individual differences in nucleus accumbens activity to food and sexual images predict weight gain and sexual behavior. J Neurosci 32:5549-5552. CrossRef Medline

Due DL, Huettel SA, Hall WG, Rubin DC (2002) Activation in mesolimbic and visuospatial neural circuits elicited by smoking cues: evidence from functional magnetic resonance imaging. Am J Psychiatry 159:954-960. Medline

Epstein LH, Truesdale R, Wojcik A, Paluch RA, Raynor HA (2003) Effects of deprivation on hedonics and reinforcing value of food. Physiol Behav 78:221-227. CrossRef Medline

Epstein LH, Leddy JJ, Temple JL, Faith MS (2007) Food reinforcement and eating: a multilevel analysis. Psychol Bull 133:884-906. CrossRef Medline

Ferreira JG, Tellez LA, Ren X, Yeckel CW, de Araujo IE (2012) Regulation of fat intake in the absence of flavour signalling. J Physiol 590:953-972. CrossRef Medline

Fields DA, Goran MI, McCrory MA (2002) Body-composition assessment via air-displacement plethysmography in adults and children: a review. Am J Clin Nutr 75:453-467. Medline 
Forman SD, Cohen JD, Fitzgerald M, Eddy WF, Mintun MA, Noll DC (1995) Improved assessment of significant activation in functional magnetic resonance imaging (fMRI): use of a cluster-size threshold. Magn Reson Med 33:636-647. CrossRef Medline

Gearhardt AN, Yokum S, Stice E, Harris JL, Brownell KD (2014) Relation of obesity to neural activation in response to food commercials. Soc Cogn Affect Neurosci 9:932-938. CrossRef Medline

Geha PY, Aschenbrenner K, Felsted J, O'Malley SS, Small DM (2013) Altered hypothalamic response to food in smokers. Am J Clin Nutr 97:1522. CrossRef Medline

Goldfield GS, Legg C (2006) Dietary restraint, anxiety, and the relative reinforcing value of snack food in non-obese women. Eat Behav 7:323-332. CrossRef Medline

Goldstein RZ, Tomasi D, Alia-Klein N, Zhang L, Telang F, Volkow ND (2007) The effect of practice on a sustained attention task in cocaine abusers. Neuroimage 35:194-206. CrossRef Medline

Haberstick BC, Smolen A (2004) Genotyping of three single nucleotide polymorphisms following whole genome preamplification of DNA collected from buccal cells. Behav Genet 34:541-547. CrossRef Medline

Hall K, Heymsfield S, Kemnitz J, Klein S, Schoeller D, Speakman J (2012) Energy balance and its components: implications for body weight regulation. J Clin Nutr 95:989-994. CrossRef Medline

Hare TA, Camerer CF, Rangel A (2009) Self-control in decision-making involves modulation of the vmPFC valuation system. Science 324:646648. CrossRef Medline

Klein A, Andersson J, Ardekani BA, Ashburner J, Avants B, Chiang MC, Christensen GE, Collins DL, Gee J, Hellier P, Song JH, Jenkinson M, Lepage C, Rueckert D, Thompson P, Vercauteren T, Woods RP, Mann JJ, Parsey RV (2009) Evaluation of 14 nonlinear deformation algorithms applied to human brain MRI registration. Neuroimage 46:786-802. CrossRef Medline

Livak KJ (1999) Allelic discrimination using fluorogenic probes and the $5^{\prime}$ nuclease assay. Genet Anal 14:143-149. CrossRef Medline

Lohman TG (1989) Assessment of body composition in children. Pediatr Exerc Sci 1:19-30.

Magarey AM, Daniels LA, Boulton TJ, Cockington RA (2003) Predicting obesity in early adulthood from childhood and parental obesity. Int J Obes Relat Metab Disord 27:505-513. CrossRef Medline

Martin LE, Holsen LM, Chambers RJ, Bruce AS, Brooks WM, Zarcone JR, Butler MG, Savage CR (2010) Neural mechanisms associated with food motivation in obese and healthy weight adults. Obesity 18:254-260. CrossRef Medline

Mundy ME, Downing PE, Honey RC, Sing KD, Graham KS, Dwyer DM (2014) Brain correlates of experience-dependent changes in stimulus discrimination based on the amount of schedule of exposure PLoS One 9:e101011. CrossRef Medline

Neville MJ, Johnstone EC, Walton RT (2004) Identification and characterization of ANKK1: a novel kinase gene closely linked to DRD2 on chromosome band 11q23.1. Hum Mutat 23:540-545. CrossRef Medline

Noonan MP, Kolling N, Walton ME, Rushworth MF (2012) Re-evaluating the role of the orbitofrontal cortex in reward and reinforcement. Eur J Neurosci 35:997-1010. CrossRef Medline

Poldrack RA, Mumford JA, Nichols TE (2011) Handbook of fMRI data analysis. Cambridge, UK: Cambridge UP.

Schultz W (2010) Dopamine signals for reward value and risk: basic and recent data. Behav Brain Funct 6:24. CrossRef Medline

Sereno MI, Huang RS (2014) Multisensory maps in parietal cortex. Curr Opin Neurobiol 24:39-46. CrossRef Medline

Singer JD (1998) Using SAS PROC MIXED to fit multilevel models, hierarchical models, and individual growth models. J Educ Behav Stat 23:323355. CrossRef
Singer JW, Willet J (2003) Applied longitudinal data analysis: modeling change and event occurrence. New York: Oxford UP.

Sladky R, Friston KJ, Tröstl J, Cunnington R, Moser E, Windischberger C (2011) Slice-timing effects and their correction in functional MRI. Neuroimage 58:588-594. CrossRef Medline

Small DM, Zatorre RJ, Dagher A, Evans AC, Jones-Gotman M (2001) Changes in brain activity related to eating chocolate: from pleasure to aversion. Brain 124:1720-1733. CrossRef Medline

Small DM, Jones-Gotman M, Dagher A (2003) Feeding-induced dopamine release in dorsal striatum correlates with meal pleasantness ratings in healthy human volunteers. Neuroimage 19:1709-1715. CrossRef Medline

Soto D, Humphreys GW, Rotshtein P (2007) Dissociating the neural mechanisms of memory-based guidance of visual selection. Proc Natl Acad Sci U S A 104:17186-17191. CrossRef Medline

Stice E, Spoor S, Bohon C, Small DM (2008a) Relation between obesity and blunted striatal response to food is moderated by TaqIA A1 allele. Science 322:449-452. CrossRef Medline

Stice E, Spoor S, Bohon C, Veldhuizen MG, Small DM (2008b) Relation of reward from food intake and anticipated food intake to obesity: a functional magnetic resonance imaging study. J Abnorm Psychol 117:924935. CrossRef Medline

Stice E, Yokum S, Bohon C, Marti N, Smolen A (2010) Reward circuitry responsivity to food predicts future increases in body mass: moderating effects of DRD2 and DRD4. Neuroimage 50:1618-1625. CrossRef Medline

Stice E, Yokum S, Burger KS, Epstein LH, Small DM (2011) Youth at risk for obesity show greater activation of striatal and somatosensory regions to food. J Neurosci 31:4360-4366. CrossRef Medline

Stice E, Yokum S, Burger K, Epstein L, Smolen A (2012) Multilocus genetic composite reflecting dopamine signaling capacity predicts reward circuitry responsivity. J Neurosci 32:10093-10100. CrossRef Medline

Stice E, Yokum S, Burger KS (2013) Elevated reward region responsivity predicts future substance use onset but not overweight/obesity onset. Biol Psychiatry 73:869-876. CrossRef Medline

Swisher JD, Halko MA, Merabet LB, McMains SA, Somers DC (2007) Visual topography of intraparietal sulcus. J Neurosci 27:5326-5337. CrossRef Medline

Tang DW, Fellows LK, Small DM, Dagher A (2012) Food and drug cues activate similar brain regions: a meta-analysis of functional MRI studies. Physiol Behav 106:317-324. CrossRef Medline

Turk MW, Yang K, Hravnak M, Sereika SM, Ewing LJ, Burke LE (2009) Randomized clinical trials of weight loss maintenance: a review. J Cardiovasc Nurs 24:58-80. CrossRef Medline

Volkow ND, Wang GJ, Telang F, Fowler JS, Thanos PK, Logan J, Alexoff D, Ding YS, Wong C, Ma Y, Pradhan K (2008) Low dopamine striatal D2 receptors are associated with prefrontal metabolism in obese subjects: possible contributing factors. Neuroimage 42:1537-1543. CrossRef Medline

Whelan R, Garavan H (2014) When optimism hurts: inflated predictions in psychiatric neuroimaging. Biol Psychiatry 75:746-748. CrossRef Medline

World Health Organization (2013) Obesity and overweight. Fact Sheet 311. Retrieved 11.10.2014 from http://amro.who.int/common/Display. asp?Lang $=\mathrm{E} \&$ RecID $=10203$.

Yokum S, Ng J, Stice E (2011) Attentional bias to food images associated with elevated weight and future weight gain: an fMRI study. Obesity 19:1775-1783. CrossRef Medline

Yokum S, Gearhardt AN, Haris JL, Brownell KD, Stice E (2014) Individual differences in striatum activity to food commercials predict weight gain in adolescents. Obesity (Silver Spring) 2014;22:2544-2551. CrossRef Medline 